\title{
Infarto de omento mayor. Presentación de dos casos con un tratamiento conservador
}

\author{
Nolberto Adrián Medina-Gallardo, ${ }^{1}$ Yuhamy Curbelo-Peña, ${ }^{1}$ Júlia Gardenyes-Martínez, ${ }^{1}$ \\ Tomás Stickar, ${ }^{1}$ Javier De Castro-Gutiérrez, ${ }^{1}$ Judit Hermoso-Bosch, ${ }^{1}$ Helena Vallverdú-Cartie ${ }^{2}$
}

${ }^{1}$ Servicio de Cirugia General. Hospital Universitario de Vic.

${ }^{2}$ Jefe de Servicio de Cirugía General. Hospital Universitario de Vic.

Vic, Barcelona, España.

Acta Gastroenterol Latinoam 2020;50(3):355-359

Recibido: 15/09/2018 / Aceptado: 14/07/2020 / Publicado online: 28/09/2020

\section{Resumen}

El infarto del omento mayor es una causa rara de dolor abdominal agudo. Casos. Presentamos el caso de un hombre de 42 años que consultó al servicio de urgencias por un dolor en el hemiabdomen derecho, con marcadores inflamatorios elevados en la analitica sanguinea. La exploración radiológica por la TC abdominal mostró un infarto omental por torsión. El paciente evolucionó favorablemente con el tratamiento conservador. El segundo caso es el de un hombre de 85 años apendicectomizado que consultó por un dolor en la fosa ilíaca izquierda; con exploración fisica y complementarios de laboratorio y radiológicos se concluye que se trata de un infarto omental, con una buena evolución a causa del tratamiento.

Correspondencia: Yuhamy Curbelo-Peña

Calle Francesc Plà, El Vigatà (C.P.: 08500), Hospital Universitario de Vic. Vic, Barcelona, España

Tel.: 0034-691-91-52-10 / Fax: 0034-93-573-09-92

Correoelectrónico: layuha@hotmail.com
Discusión. La torsión omental primaria se produce cuando un segmento móvil, espeso de omento, gira alrededor de un punto fijo proximal en ausencia de una patología intraabdominal. Por otro lado, la torsión secundaria se asocia con una serie de condiciones preexistentes, siendo la más común la hernia inguinal. Clinicamente puede imitar un cuadro de apendicitis o colecistitis aguda, por lo que debe tenerse en cuenta en el diagnóstico diferencial. Si bien la laparoscopia puede ayudar en el diagnóstico y en el tratamiento, se puede evitar la cirugía de manera segura después del diagnóstico radiológico, con excelentes resultados en el seguimiento. Conclusión. El infarto omental en una entidad importante en el diagnóstico diferencial del abdomen agudo, con una crucial importancia de la TC abdominal para su confirmación, evitando así la intervención quirúrgica, la cual podría no ser necesaria.

Palabras claves. Infarto omental, torsión omental, tratamiento conservador.

\section{Infarction of Greater Omentum. Two cases with conservative treatment}

\section{Summary}

Omental infarction is a rare cause of acute abdominal pain. Cases. The report is based on two cases who attended to emergency, complaining of abdominal pain with CT diagnosis of omental infarction. We present a case of a 42 years old man who consulted to the emergency service for a 
pain in the right hemiabdomen, with elevated inflammatory markers in blood tests. Radiological examination by abdominal CT showed an omental torsion infarction. The patient evolved favourably with conservative treatment. The second case is a 85 years old man appendectomized who consulted for a pain in the left iliac foza with physical examination and complementary and radiological, it is concluded that it is an omental infarction, with a good evolution due to the treatment. Discussion. Clinical diagnosis remains a challenge, due to its rarity and clinical presentation mimicking acute appendicitis or cholecystitis. Hence in the absence of imaging test, intraoperative diagnosis takes place. It can occur due to two main pathogenic mechanisms: Secondary to vascular pedicle torsion (primary or secondary to another abdominal pathology) or situations that predispose to thrombosis. Conclusion. Omental infarction should be considered in the differential diagnosis of acute abdominal pain. Once confirmed by CT abdominal scan, conservative treatment could be considered, avoiding unnecessary surgery.

Key words. Omentum infarction, omental torsion, conservative treatment.

\section{Abreviaturas}

IO: Infarto omental.

TC: Tomografia computarizada.

\section{Introducción}

El infarto omental (IO) es una causa poco frecuente de dolor abdominal agudo cuyo diagnóstico se basa actualmente en hallazgos intraoperatorios o radiológicos. Afecta, en general, la porción derecha omental por lo que el diagnóstico a menudo se realiza durante cirugías por una sospecha diagnóstica de apendicitis o colecistitis. En el caso del diagnóstico preoperatorio por técnicas radiológicas, su manejo posterior es controvertido. La experiencia acumulada se basa principalmente en publicaciones de casos clínicos aislados donde se ha optado tanto por el manejo conservador como por el tratamiento quirúrgico de entrada. Presentamos el caso de dos pacientes con diagnóstico de infarto omental por torsión primaria en los cuales se decidió optar por tratamientos conservadores. Además, realizamos una revisión a propósito de esta infrecuente patología.

\section{Caso 1}

El paciente es de sexo masculino y tiene 42 años de edad. No posee antecedentes patológicos ni cirugías previas. Consultó en el Servicio de Urgencias por un dolor abdominal de 48 horas de evolución en el hemiabdomen derecho, acompañado de náuseas y dos deposiciones disminuidas de consistencia. En la exploración física presentó una temperatura de $37,4^{\circ} \mathrm{C}$ y sensación de una ocupación dolorosa en el hemiabdomen derecho, sin signos de irritación peritoneal, en un paciente sin afectación del estado general. En la analítica sanguínea se destacó una leucocitosis de 14500 sin desviación a formas inmaduras y una PCR de 123. Se le solicitó una ecografía abdominal por sospechar que se trataba de una probable colecistitis, evidenciando un engrosamiento con hiperecogenicidad patológica de la grasa en el hemiabdomen derecho, con líquido libre y asas edematosas. En la TC abdominal se objetivó, además, una imagen de "remolino vascular" (Figuras 1, 2 y 3), hallazgos compatibles con un infarto omental por torsión, acompañado de un edema mural de una extensa porción del íleon.

Dados los hallazgos de la TC, a nivel intestinal se le realizó una entero-RM con gadolinio que descartó un proceso intestinal inflamatorio subyacente. Se decidió un tratamiento conservador con dieta absoluta y analgesia endovenosa. El curso clínico fue favorable, recibiendo el alta al cuarto día de haber ingresado. Se le realizó el seguimiento ambulatorio al mes, encontrándose el paciente asintomático.

Figura 1. Corte coronal de la TC abdominal donde se observan los cambios en el hemiabdomen derecho que diagnostican el infarto omental

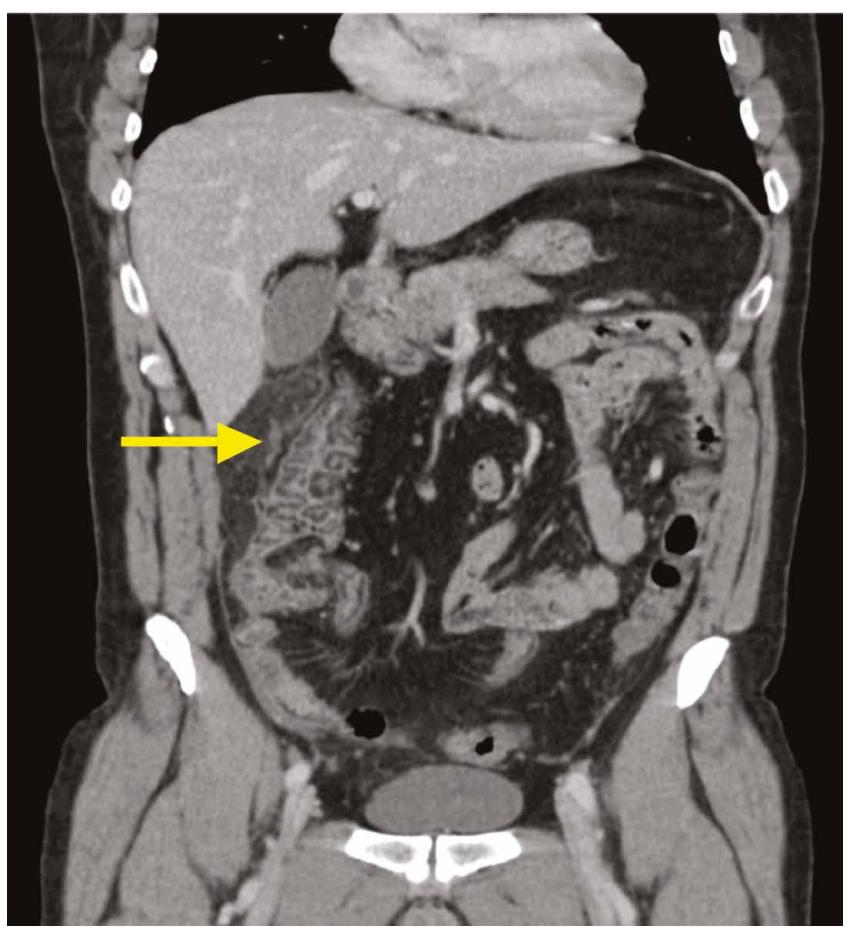


Figura 2. Corte transversal de la TC abdominal donde se observan los cambios en el hemiabdomen derecho que diagnostican el infarto omental

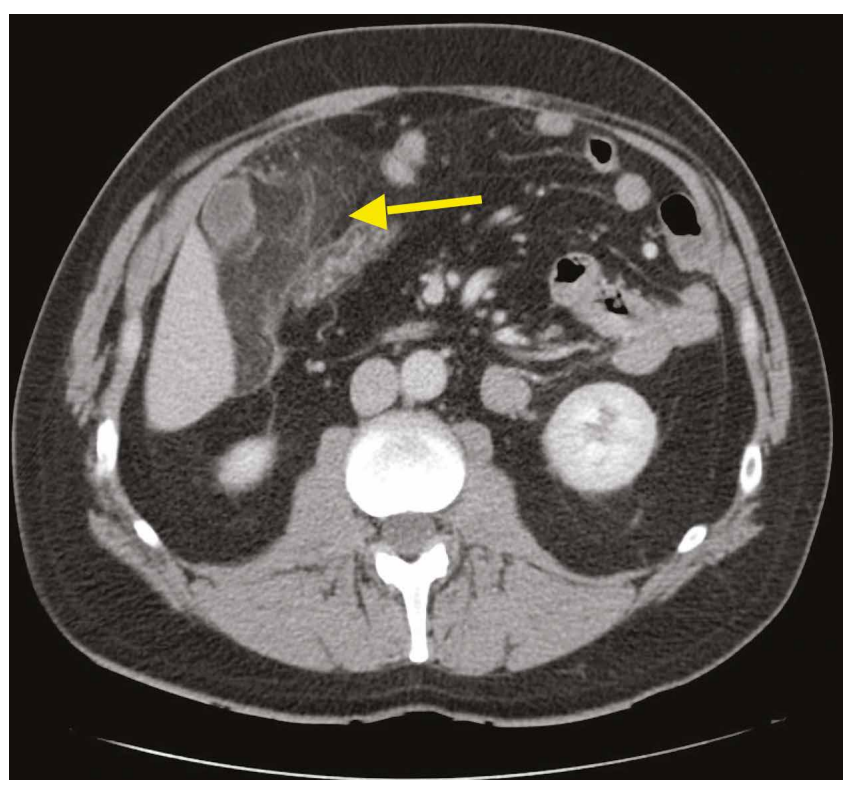

\section{Caso 2}

El paciente de sexo masculino de 85 años apendicectomizado presenta un antecedente de prostatismo. Consultó en el Servicio de Urgencias por un dolor abdominal de 7 días de evolución en la fosa ilíaca derecha, acompañado de anorexia, sin nauseas ni vómitos. En la exploración física estaba afebril, con dolor en la palpación profunda en la fosa ilíaca izquierda, sin signos de irritación peritoneal. En la analítica sanguínea presentó 7400 leucocitos sin desviación a formas inmaduras y una PCR de 29,9. Con sospecha de una diverticulitis aguda, se le solicitó una TC abdominal, objetivándose un incremento de la densidad de la grasa de 4,5 $\mathrm{cm}$ de diámetro con un halo hiperdenso periférico adyacente a la pared abdominal anterior, compatible con un infarto omental sin otros hallazgos. El diagnóstico diferencial con la apendagitis epiploica se consideró, pero en la revisión de las imágenes por el Departamento de Radiología se mantuvo el diagnóstico de infarto omental. El tratamiento conservador se decidió con una dieta absoluta y analgesia endovenosa. El curso clínico transcurrió sin incidentes y el paciente fue dado de alta el segundo día de haber ingresado. En el seguimiento un mes después, permaneció asintomático.

\section{Discusión}

Descrito por primera vez por Eitel en 1899, el IO es un cuadro poco frecuente del que solo alrededor de 300
Figura 3. Corte transversal de la TC abdominal donde se observa un enrarecimiento o aumento de grasa omental en el hemiabdomen derecho, sugestiva de un infarto omental

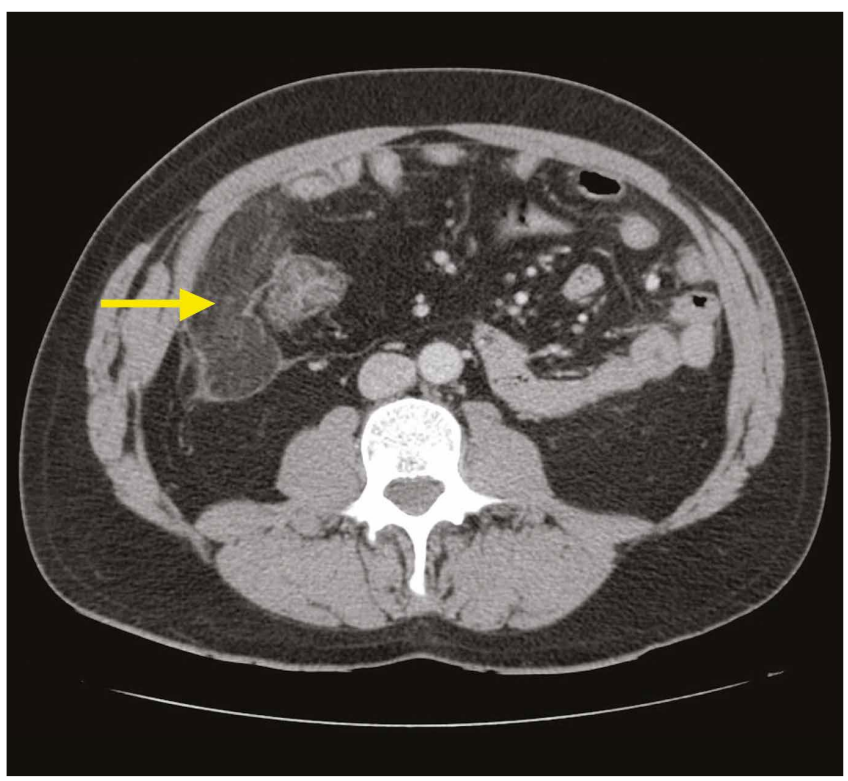

casos han sido publicados. ${ }^{1,2}$ El diagnóstico clínico es difícil por la ausencia de pruebas complementarias, dada la rareza de su aparición y la similitud clínica con otros cuadros agudos intraabdominales más frecuentes como la colecistitis, la apendicitis o la diverticulitis, lo que lleva en ocasiones a que su diagnóstico sea intraoperatorio, sobre todo en el caso de las dos primeras. ${ }^{3-6}$

El infarto omental puede ocurrir por dos mecanismos patogénicos principales: como consecuencia de una torsión de su pedículo vascular sobre su propio eje (habitualmente en el sentido de las agujas del reloj), o debido a situaciones que predispongan a la trombosis como los estados de hipercoagulabilidad o las anomalías vasculares. En consecuencia, en ambas circunstancias se ve comprometida la vascularización, resultando en una extravasación hemorrágica, con producción de líquido sanguinolento, la necrosis y la formación de adherencias. ${ }^{7}$

Las torsiones se pueden dividir en primarias o secundarias: las primeras no tienen patología subyacente; en las segundas, que son responsables de aproximadamente dos tercios de los casos, ${ }^{7}$ hay una presencia de un proceso patológico que hace de punto de "anclaje" distal del omento (los quistes, los tumores, los focos inflamatorios intrabdominales, las heridas quirúrgicas previas o los sacos herniarios con epiplón en su interior). ${ }^{8}$ Son más frecuentes del lado derecho ( $90 \%$ de los casos), en principio debido al tamaño superior y movilidad del epiplón mayor de ese 
lado con respecto al izquierdo, ${ }^{9}$ con el punto de torsión alrededor de la arteria epiploica derecha distal.

Se han descrito a los factores desencadenantes como los traumatismos de la pared abdominal, la tos, los ejercicios o trabajos intensos, el aumento del peristaltismo, los purgantes o las maniobras de reducción herniaria. En cuanto a la edad de aparición se ha presentado desde la edad pediátrica ${ }^{10-13}$ hasta en pacientes ancianos, ${ }^{8}$ si bien la mayoría de los casos aparece en personas entre los 30 y 50 años, con predominio en el sexo masculino, sobre todo obesos. ${ }^{14}$

Habitualmente los pacientes se presentan con un dolor abdominal continuo, no irradiado, cuya intensidad va en aumento. La presencia de las náuseas y los vómitos es variable. ${ }^{10}$ Aproximadamente la mitad puede presentar febrícula y discreta leucocitosis en la analítica. Si bien la mayoría se presenta como un único episodio de dolor abdominal, algunos pacientes pueden sufrir un dolor recurrente, lo cual puede sugerir torsiones intermitentes. La sospecha diagnóstica inicial, de forma habitual, apunta a una apendicitis, una colecistitis, un quiste ovárico complicado en el caso de la fosa ilíaca derecha, ${ }^{15}$ una diverticulitis con dolor contralateral y una adenitis mesentérica o un divertículo de Meckel complicado, en el caso de la población pediátrica. En general, los pacientes con una torsión omental presentan menos afectación del estado general y una menor cantidad de signos de respuesta inflamatoria que otros procesos abdominales con el mismo tiempo de evolución. ${ }^{7}$

La ecografía abdominal puede ser de utilidad sobre todo para descartar otras causas de abdomen agudo. Si bien para el diagnóstico de infarto omental presenta menor rendimiento, con frecuencia se observa una masa intraabdominal hiperecoica no compresible. ${ }^{8,}{ }^{15}$ Con el incremento del uso de la TC abdominal, el diagnóstico preoperatorio de esta patología ha ido en aumento, lo que ha llevado al mismo tiempo a un aumento del manejo conservador, ${ }^{7,19,23}$ con buenos resultados, siendo raras las complicaciones evolutivas. ${ }^{22}$ En este estudio, una masa bien delimitada de grasa, entre la pared abdominal y el colon, 14 las imágenes lineales concéntricas en forma de remolino y la presencia de un pedículo hipocaptante ayudan a realizar el diagnóstico. ${ }^{1}$

Aunque es considerada una patología benigna y de naturaleza autolimitada que puede ser tratada de una forma conservadora con excelentes resultados hasta en un $85 \%$ de los casos, ${ }^{19}, 22$ algunos autores propugnan la exploración quirúrgica de entrada incluso con diagnóstico tomográfico., 7 Se argumenta a favor de la cirugía una rápida resolución del cuadro doloroso, una menor estancia hospitalaria y una pronta reincorporación a la vida habitual, ${ }^{16}$ evitando complicaciones evolutivas como la formación de colecciones y las adherencias.

Varias de las publicaciones recientes presentan a la cirugía laparoscópica como la herramienta definitiva para el diagnóstico y el manejo de esta patología. 1, 7, 8, 14, 17, 20, 25 Sin embargo, puede ser necesaria una laparotomía media si no se consigue la resolución por la vía menos invasiva o en caso de necesitar realizar resecciones extensas del omento. ${ }^{11,18,21}$

En nuestro caso, la historia de corto tiempo de evolución (48 horas) con la ocupación dolorosa en el hemiabdomen derecho nos decidió por la realización de pruebas de imagen ante la sospecha de un proceso más evolucionado, con los resultados comentados.

Finalmente, la aparición de las asas reactivas de aspecto inflamatorio también ha sido descrita, ${ }^{19}$ aunque en nuestro caso la inflamación primaria intestinal fue descartada mediante entero-RM.

\section{Conclusión}

El IO debe ser incluido en el espectro de los diagnósticos diferenciales de dolor abdominal agudo en los pacientes con poca afectación del estado general y ante la aparición de una "ocupación" o masa. En estos casos, el uso de la TC es la mejor prueba para su diagnóstico ya que permite, además, descartar otras patologías. Si bien el abordaje laparoscópico es atractivo, el tratamiento conservador debe ser considerado en los casos de un diagnóstico positivo en las pruebas de imagen, ya que evita un tratamiento quirúrgico que, según la evidencia disponible, puede en algunos casos ser innecesario. Consideramos que esta serie de casos aporta a la literatura una herramienta actualizada para el manejo de esta patología.

Agradecimientos. A todo el equipo de trabajo del Servicio de Cirugia General del Hospital Universitario de Vic, por su desempeño y labor.

Conflicto de intereses. Los autores declaran no tener ningún conflicto de interés potencial relevante para el articulo de ninguna indole.

Fuente de financiación. Ninguna para este trabajo.

\section{Referencias}

1. Alexiou K, Ioannidis A, Drikos I, Sikalias N, Economou N. Torsion of the greater omentum: two case reports. J Med Case Rep 2015; 9: 160 .

2. Charieg A, Ben Ahmed Y, Nouira F, Channoufi F, Jouini R, Jlidi S. A diagnosis to keep in mind: primary omental torsion in children. EC Paediatrics 2016; 2: 245-249. 
3. Dhooghe V, Reynders D, Cools P. Torsion of a bifid omentum as a rare cause of acute abdomen: a case report. J Med Case Rep 2016; 10: 289.

4. Zaleta-Cruz JL, Rojas-Méndez J, Garza-Serna U, González-Ruvalcaba R, Ortiz de Elguea-Lizarraga J, Flores-Villalba E. Omental torsion. Case Report. Cir Cir 2017; 85: 49-53.

5. Smolilo D, Lewis BC, Yeow M, Watson DI. Omental infarction mimicking cholecystitis. Case Rep Surg 2015; 1: 1-3.

6. Tasleem A, Zaman Q, Thomas DA, Payne JG, Kerwat R, Khan AA. Omental torsion: an unusual cause of right iliac fossa pain and role of laparoscopic management. Gastroenterology Res 2013; 6: 237-239.

7. Breunung N, Strauss P. A diagnostic challenge: primary omental torsion and literature review - a case report. World J Emerg Surg 2009; 4: 40.

8. Cremonini C, Bertolucci A, Tartaglia D, Menonna F, Galatioto C, Chiarugi M. Acute abdomen caused by greater omentum torsion: A case report and review of the literature. Ulus Travma Acil Cerrahi Derg 2016; 22: 391-394.

9. López-Rubio MA, Martínez-Ruiz Y. Una causa infrecuente de dolor abdominal: el infarto de omento. Rev Clin Med Fam 2011; 4: 254-255.

10. Occhionorelli S, Zese M, Cappellari L, Stano R, Vasquez G. Acute Abdomen due to Primary Omental Torsion and Infarction. Case Rep in Surg 2014; 2014: 1-4.

11. Mendoza Moreno F, Díez Gago MR, Córdova García DM, Pedraza Muñoz A, Díez Alonso M, Noguerales Fraguas F, GranellVicent FJ. Primary omental torsion as presentation of acute abdomen. Case report. Rev Esp Enferm Dig 2016; 108: 105-106.

12. Andreuccetti J, Ceribelli C, Manto O, Chiaretti M, Negro P, Tuscano D. Primary omental torsion (POT): a review of literature and case report. World J Emerg Surg 2011; 6: 6.

13. Joshi S, Cuthbert GA, Kerwat R. Omental torsion, a rare cause of acute abdomen. BMJ Case Rep 2016; 1: 1-3.
14. Vagholkar K, Chougle Q, Agrawal P, Daga D, Vagholkar S. Omental torsion: a rare cause of acute abdomen. Int Surg J 2016; 3: 1711-1713.

15. Raza N, Kania P, Bhamare P. A rare case of omental torsion - a surprise diagnosis of acute pelvic pain. Int J Reprod Contracept Obstet Gynecol 2016; 5: 1-3.

16. Sánchez Fuentes PA, López López V, Febrero B, Ramírez P, Parrilla Paricio P. Infarto omental: ¿manejo quirúrgico o conservador? Cir Esp 2015; 93: 475-477.

17. Ghosh Y, Arora R. Omental torsion. JCDR 2014; 8: 1-2.

18. Katagiri H, Honjo K, Nasu M, Fujisawa M, Kojima K. Omental Infarction due to Omental Torsion. Case Rep Surg 2010; 1: 1-3.

19. Lapsia S, Ghai S. Omental infarction: a rare cause of acute abdominal pain. Emerg Med J 2007; 24: 779.

20. AbdulAziz A, El Zalabany T, Al Sayed AR, Al Ansari A. Idiopathic omental infarction, diagnosed and managed laparoscopically: a case report. Case Rep Surg 2013; 2013: 193546.

21. Scabini S, Rimini E, Massobrio A, Romairone E, Linari C, Scordamaglia R, Marini LD, Ferrando V. Primary omental torsion: A case report. World J Gastrointest Surg 2011; 3: 153-155.

22. Soobrah R, Badran M, Smith SG. Conservative management of segmental infarction of the greater omentum: a case report and review of literature. Case Rep Med 2010; 2010: 1-4.

23. Fernández-Rey CL. Infarto omental primario como causa de abdomen agudo no quirúrgico: diagnóstico por imagen. Rev Esp Enferm Dig 2010; 102: 498-499.

24. Sierra P, Cabrera R, Fuentealba I, Soto G, Abud M. Caso clínico radiológico para diagnóstico. Rev chil Radiol 2009; 15: 155-158.

25. Kerem M, Bedirli A, Mentes BB, Sakrak O, Pala I, Oguz M. Torsion of the greater omentum: preoperative computed tomographic diagnosis and therapeutic laparoscopy. JSLS 2005; 9: 494-496. 